\title{
DIGITALCOMMONS
}

@WAYNESTATE -

Wayne State University

$1-1-2015$

\section{Almost Sure Asymptotic Stabilization of Differential Equations with Time-Varying Delay by Lévy Noise}

Dezhi Liu

Nanjing University of Science and Technology

Weiqun Wang

Nanjing University of Science and Technology

Jose Luis Menaldi

Wayne State University, menaldi@wayne.edu

\section{Recommended Citation}

Liu, D., Wang, W. \& Menaldi, J.L. Nonlinear Dyn (2015) 79: 163. doi: 10.1007/s11071-014-1653-1

Available at: https://digitalcommons.wayne.edu/mathfrp/57

This Article is brought to you for free and open access by the Mathematics at DigitalCommons@WayneState. It has been accepted for inclusion in Mathematics Faculty Research Publications by an authorized administrator of DigitalCommons@WayneState. 


\title{
Almost sure asymptotic stabilization of differential equations with time-varying delay by Lévy noise*
}

\author{
Dezhi Liu ${ }^{a, b, c \dagger}$ Weiqun Wang ${ }^{a}$, Jose Luis Menaldi ${ }^{c}$ \\ ${ }^{a}$ Department of Applied Mathematics,Nanjing University of Science and Technology, \\ Nanjing, Jiangsu 210094, China \\ ${ }^{b}$ School of Statistics and Applied Mathematics,Anhui University of Finance and Economics, \\ Bengbu, Anhui 233030, China \\ ${ }^{c}$ Department of Mathematics, Wayne State University, Detroit, MI, 48202, USA
}

\begin{abstract}
This paper aims to determine that the Lévy noise can stabilize the given differential equations with time-varying delay,which has generalized the Brownian motion case. An analysis is developed and sufficient conditions on the stabilization for stochastic differential equations with time-varying delay are presented.Our stabilization criteria is in terms of linear matrix inequalities (LMIs), whence the feedback controls can be designed more easily in practice.
\end{abstract}

Keywords: stabilization; Lévy noise; almost surely asymptotically stable; stochastic differential equations; LMIs.

2000 Mathematics Subject Classification: 60H10, 93D15, 37H10.

\section{Introduction}

In the past decades, the problems of stabilization synthesis for stochastic systems have received significant attentions, and many results have been reported (see, e.g., Appleby et al.[3], Deng et al.[9], Hu\&Mao[10], Hu et al.[11], Huang[12], Li\&De Souza[15], Shen et al.[23], Xie et al.[25] and Yue\&Han[29]). Generally, the stabilization problems can be solved in the moment sense (see, e.g., Liu et al.[17] and Wei et al.[24]), however, in the recent years, the moment sense has not met the need of control theory, the almost sure sense of stabilization problems for stochastic systems have been focused on (see, e.g., Bercu et al.[8], Huang\&Mao[13], Liu et al.[17], Mao[18] and Mao et al. $[20])$.

In the above stabilization problems, it is well known that an unstable deterministic dynamical system can be stabilized when it is perturbed by noise. There have been a number of studies of the topic using different types of noise and the following paper list is far from exhaustive (see, e.g.,

${ }^{*}$ This work was partially supported by PNSF of Anhui (1208085QG131,KJ2013Z008), SRFDP of China (20133219110040), NNSF of China (11301001), HSSF for the Ministry of Education(10YJC630143), EYSF of Anhui Province of China (2013SQRL030ZD).

${ }^{\dagger}$ Corresponding author, E-mail address: mathliudz@163.com (D.Liu). 
Arnold\&Crauel[4], Bellman et al.[7], Khasminski[14] and Mao[19]), in these sources of noise, the mutli-dimensional Brownian motion has be recognized as the general theory of stochastic stabilization by Mao[19]. Furthermore,the Lévy process as the source of noise has been employed to stabilize the unstable dynamical system, which is the more general theory and builds extensively on Mao's results in the Brownian motion case, and less studies seem to be timely as there has recently been extensive activity from the point of view of both theoretical development and applications of Lévy processes (see, e.g., Applebaum\&Siakalli[2], Bao and Yuan[5]).

On the other hand, time delays are frequently encountered in a variety of dynamic systems, such as nuclear reactors, chemical engineering systems, biological systems, and population dynamics models (see, e.g., Bao and Yuan[6], Hu et al.[11], Huang\&Mao[13], Yang et al.[27]). They are often a class of source of instability and poor performance of systems. So the problems of stabilization synthesis of differential equations with time-varying delay have become more important and interesting, the exist efforts can be classified into two aspects, the moment sense criteria (see, e.g., Liu et al.[16], Mao et al.[21], Zohrabi et al.[30]) and the almost sure sense criteria (see, e.g., Huang\&Mao[13], Yang et al.[27] and Yuan\&Mao[28]).

However, to the authors' best knowledge, when the stabilization of differential equations with time-varying delays are considered, the almost surely asymptotically stable analysis of differential equations with time-varying delay by Lévy noise have not been adequately addressed and remain to be an interesting and challenging research topic, which are dealt with in this paper, and the purpose of stabilization is to develop conditions such that the underlying systems are almost surely asymptotically stable. Following the same idea as in dealing with the stabilization problem, linear state feedback controllers are designed such that the nonlinear closed-loop systems are almost surely asymptotically stable. In order to design easily in practice, the explicit expressions for the desired state feedback controllers are given with LMIs. Therefore, the main contribution of this paper are mainly twofold: (1)The source of noise for almost sure asymptotic stabilization is Lévy process; (2)The explicit expressions for the desired state feedback controllers are clear with LMIs, which is easy to be designed.

The rest of the paper is organized as follows. In section 2, we present some basic preliminaries and the form of differential equations with time-varying delay and Lévy process. In section 3, the useful lemma of global solution analysis and the definition are presented, and the sufficient conditions for stabilization of differential equations with time-varying delay by Lévy noise have been given, a simple example is also presented. In section 4 , the desired state feedback controllers are designed with LMIs under the useful lemma. In section 5, the related discussion on the main results have been presented.

\section{Preliminaries}

Let $\left\{\Omega, \mathcal{F},\left\{\mathcal{F}_{t}\right\}_{t \geq 0}, \mathbf{P}\right\}$ be a complete probability space with a filtration satisfying the usual conditions, i.e., the filtration is continuous on the right and $\mathcal{F}_{0}$ contains all P-zero sets. Let $D\left([-\tau, 0] ; R^{n}\right)$ denote the family of functions $\varphi$ from $[-\tau, 0]$ to $R^{n}$ that are right-continuous and have limits on the left. $D\left([-\tau, 0] ; R^{n}\right)$ is equipped with the norm $\|\varphi\|=\sup _{-\tau \leq s \leq 0}|\varphi(s)|$ and $|x|=\sqrt{x^{T} x}$

for any $x \in R^{n}$. If $\mathrm{A}$ is a vector or matrix, its trace norm is denoted by $|A|=\sqrt{\operatorname{trace}\left(A^{T} A\right)}$, while its operator norm is denoted by $\|A\|=\sup \{|A x|:|x|=1\}$. Denote by $D_{\mathcal{F}_{0}}^{b}\left([-\tau, 0] ; R^{n}\right)$ the family of all bounded, $\mathcal{F}_{0}$ measurable, $D\left([-\tau, 0] ; R^{n}\right)$-valued random variables. We denote by $L^{1}\left(R_{+} ; R_{+}\right)$ 
the family of all functions $\lambda(t)$ such that $\int_{0}^{\infty} \lambda(t) d t<\infty$, and denote by $\mathcal{K}\left(R_{+} ; R_{+}\right)$the family of all functions $\gamma(x)$, if it is continuous, strictly increasing and $\gamma(0)=0$. It is said to belong to the family $\mathcal{K}_{\infty}$, if $\gamma \in \mathcal{K}$ and $\gamma(x) \rightarrow \infty$ as $x \rightarrow \infty$.

Let us begin with the discussion the stabilization of the differential equation with time-varying delay by the Lévy process

$$
\dot{x}(t)=f(t, x(t), x(t-\tau(t)))
$$

where $\tau$ and $d_{\tau}$ are positive constants and $\tau(t)$ is nonnegative differential function which denotes the time-varying delay and satisfies

$$
0 \leq \tau(t) \leq \tau, \dot{\tau}(t) \leq d_{\tau}<1
$$

Consider the following stabilized differential equations with time-varying by Lévy process

$$
d x(t)=f\left(t, x\left(t^{-}\right), x\left(t^{-}-\tau\left(t^{-}\right)\right)\right) d t+u\left(x\left(t^{-}\right)\right) d Y(t)
$$

with initial value $x_{0}=\{x(\theta):-\tau \leq \theta \leq 0\}=\xi \in D_{\mathcal{F}_{0}}^{b}\left([-\tau, 0] ; R^{n}\right)$, where $(Y(t), t \geq 0)$ is a Lévy process taking values in $R^{m}, u(x(t))$ is the feedback control, and we consider the feedback control is linear and the form is as follows,

$$
u(x(t))=\left(K_{1} x(t), K_{2} x(t), \ldots, K_{m} x(t)\right),
$$

where $\left(K_{i}, i=1,2, . ., m\right)$ are controller parameters to be designed, then the system (2.2) becomes

$$
d x(t)=f\left(t, x\left(t^{-}\right), x\left(t^{-}-\tau\left(t^{-}\right)\right)\right) d t+\sum_{i=1}^{m} K_{i} x\left(t^{-}\right) d Y_{i}(t) .
$$

In order to give the Lévy-Itô decomposition of $(Y(t), t \geq 0)$, we present the following assumption I, which holds in the rest paper:

\section{Assumption I}

$$
\int_{R^{m} \backslash\{0\}}\left(|y|^{2} \wedge 1\right) \pi(d y)<\infty .
$$

So the Lévy process $(Y(t), t \geq 0)$ has the following decomposition

$$
Y_{i}(t)=b_{i} t+B_{i}^{A}(t)+\int_{|y|<1} y_{i} \tilde{N}(t, d y)+\int_{|y| \geq 1} y_{i} N(t, d y)
$$

where $B^{A}(t)$ is an m-dimensional Brownian motion with covariance matrix $\mathrm{A}$, and $B_{i}^{A}(t)=\sum_{j=1}^{p} \sigma_{i j} B_{j}(t)$, $B_{1}, B_{2}, . ., B_{p}$ are standard Brownian motion, and $\sigma_{A}=\left(\sigma_{i j}\right)_{m \times p}$ is a real-valued matrix for which

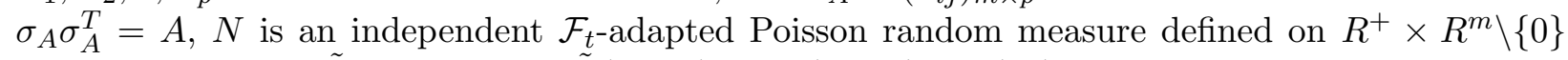
with compensator $\tilde{N}$ of the form $\tilde{N}(d t, d y)=N(d t, d y)-\pi(d y) d t$, where $\pi$ is a Lévy measure. Note that if for some $p \geq 1, E|Y(t)|^{p}<\infty$ for all $t \geq 0$, then $\int_{|y| \geq 1}|y|^{p} \pi(d y)<\infty$ and hence Lévy process $(Y(t), t \geq 0)$ admits the following decomposition

$$
Y_{i}(t)=\tilde{b}_{i} t+B_{i}^{A}(t)+\int_{R^{m} \backslash\{0\}} y_{i} \tilde{N}(t, d y) .
$$


Therefore, (2.3) can be rewritten as

$$
\begin{aligned}
d x(t)=\left(f\left(t, x\left(t^{-}\right), x\left(t^{-}-\tau\left(t^{-}\right)\right)\right)+\sum_{i=1}^{m} K_{i} x\left(t^{-}\right) \tilde{b}_{i}\right) d t & +\sum_{j=1}^{p} K_{j}^{*} x\left(t^{-}\right) d B_{j}(t) \\
& +\sum_{i=1}^{m} \int_{R^{m} \backslash\{0\}} K_{i} x\left(t^{-}\right) y_{i} \tilde{N}(d t, d y)
\end{aligned}
$$

where $K_{j}^{*}=\sum_{i=1}^{m} K_{i} \sigma_{i j}$ and $f: R_{+} \times R^{n} \times R^{n} \rightarrow R^{n}$ satisfies the local Lipschitz condition:

Assumption II Local Lipschitz condition For any $k=0,1,2, \ldots$, there exists $L_{k}>0$, such that

$$
\left|f\left(t, x_{1}, x_{2}\right)-f\left(t, \bar{x}_{1}, \bar{x}_{2}\right)\right| \leq L_{k}\left(\left|x_{1}-\bar{x}_{1}\right|+\left|x_{2}-\bar{x}_{2}\right|\right)
$$

where $t \in R_{+}, x_{1}, x_{2}, \bar{x}_{1}, \bar{x}_{2} \in R^{n}$, and $\left|x_{1}\right| \vee\left|x_{2}\right| \vee\left|\bar{x}_{1}\right| \vee\left|\bar{x}_{2}\right| \leq k$, which guarantee the uniqueness and existence of the local solution for system (2.6). We also assume that $f(t, 0,0)=0$, then the system (2.6) has a trivial solution $x(t) \equiv 0$ for all $t \geq 0$ with initial condition $x_{0}=\xi=0$, the nosie perturbation preserves the equilibrium of the system (2.6).

Furthermore, we will give the sufficient conditions, which guarantee the system (2.6) has a global solution under the Assumption III in the next section.

Assumption III Assume that $V \in C^{1,2}\left(R_{+} \times R^{n} ; R_{+}\right), \lambda_{1} \in L^{1}\left(R_{+} ; R_{+}\right)$, and $\mu_{1}, \mu_{2}: R^{n} \rightarrow R_{+}$ are continuous and nonnegative, for any $(t, x, \bar{x}) \in R_{+} \times R^{n} \times R^{n}$,

$$
L V(t, x, \bar{x}) \leq \lambda_{1}(t)-\mu_{1}(x)+\mu_{2}(\bar{x}), \mu_{1}(0)=\mu_{2}(0)=0, \mu(x)=\mu_{1}(x)-\mu_{2}(x)>0, \forall x \neq 0,
$$

where $L$ is the differential operator, which is associated with equation (2.6) and acts on the $V$ function, then

$$
\begin{aligned}
& L V(t, x, \bar{x}) \\
& =V_{t}(t, x)+V_{x}(t, x)\left[f(t, x, \bar{x})+\sum_{i=1}^{m} K_{i} x \tilde{b}_{i}\right]+\frac{1}{2} \sum_{j=1}^{p}\left[\left(K_{j}^{*} x\right)^{T} V_{x x}(t, x)\left(K_{j}^{*} x\right)\right] \\
& \quad+\sum_{i=1}^{m} \int_{R^{m} \backslash\{0\}}\left[V\left(t, x+K_{i} x y_{i}\right)-V(t, x)-V_{x}(t, x) K_{i} x y_{i}\right] \pi(d y)
\end{aligned}
$$

where

$$
\begin{gathered}
V_{t}(t, x)=\frac{\partial V(t, x)}{\partial t}, V_{x}(t, x)=\left(\frac{\partial V(t, x)}{\partial x_{1}}, \ldots, \frac{\partial V(t, x)}{\partial x_{n}}\right) \\
V_{x x}(t, x)=\left(\frac{\partial^{2} V(t, x)}{\partial x_{i} \partial x_{j}}\right)_{n \times n} .
\end{gathered}
$$




\section{Global solution analysis and almost sure asymptotic stabiliza- tion by Lévy noise}

In this section, at first, we consider the global topic of the solution to system (2.6), by virtue of the local Lipschitz condition, we know that there exists a unique adapted process $x$ such that

$$
\begin{aligned}
x\left(t \wedge \rho_{k}\right)=\xi & +\int_{0}^{t \wedge \rho_{k}}\left(f\left(s, x\left(s^{-}\right), x\left(s^{-}-\tau\left(s^{-}\right)\right)\right)+\sum_{i=1}^{m} K_{i} x\left(s^{-}\right) \tilde{b}_{i}\right) d s \\
& +\sum_{j=1}^{p} \int_{0}^{t \wedge \rho_{k}} K_{j}^{*} x\left(s^{-}\right) d B_{j}(s)+\sum_{i=1}^{m} \int_{0}^{t \wedge \rho_{k}} \int_{R^{m} \backslash\{0\}} K_{i} x\left(s^{-}\right) y_{i} \tilde{N}(d s, d y), \quad t \geq 0, \text { a.s. }
\end{aligned}
$$

where $\rho_{k}=\inf \{t>0:|x(t ; \xi)| \geq k, \xi \neq 0\}$, and set $\inf \emptyset=\infty$ as usual. If the explosion time $\rho_{e}$ satisfies $\rho_{e}=\infty$ a.s., then the system (2.6) has a global solution, where $\rho_{e}$ defined as follow,

$$
\rho_{e}=\lim _{k \rightarrow \infty} \rho_{k}=\inf \{t>0:|x(t ; \xi)| \notin[0, \infty)\},
$$

with local solution theory, let us present the following lemma for global solution and the definition of the almost sure asymptotic stability for the system (2.6).

Lemma 3.1. Under the Assumption II and III, and $V(t, x)$ is decrescent radially unbounded, i.e.

$$
\lim _{|x| \rightarrow \infty} \inf _{0 \leq t<\infty} V(t, x)=\infty .
$$

Then for any initial value $\{x(\theta):-\tau \leq \theta \leq 0\}=\xi \in D_{\mathcal{F}_{0}}^{b}\left([-\tau, 0] ; R^{n}\right)$, the system (2.6) has a unique global solution.

Proof. Using the Itô formula for the system (2.6), we obtain, for any $t \geq 0$

$$
\begin{aligned}
V(t, x(t))= & V(0, \xi)+\int_{0}^{t} L V\left(s, x\left(s^{-}\right), x\left(s^{-}-\tau\left(s^{-}\right)\right)\right) d s+\sum_{j=1}^{p} \int_{0}^{t} V_{x}\left(s, x\left(s^{-}\right)\right) K_{j}^{*} x\left(s^{-}\right) d B_{j}(s) \\
& +\sum_{i=1}^{m} \int_{0}^{t} \int_{R^{m} \backslash\{0\}}\left[V\left(s, x\left(s^{-}\right)+K_{i} x\left(s^{-}\right) y_{i}\right)-V\left(s, x\left(s^{-}\right)\right)\right] \tilde{N}(d s, d y) .
\end{aligned}
$$

Due to the optional sampling theorem and (2.7), we get

$$
\begin{aligned}
& E V\left(t \wedge \rho_{k}, x\left(t \wedge \rho_{k}\right)\right) \\
& =V(0, \xi)+E \int_{0}^{t \wedge \rho_{k}} L V\left(s, x\left(s^{-}\right), x\left(s^{-}-\tau\left(s^{-}\right)\right)\right) d s \\
& \leq V(0, \xi)+\int_{0}^{t} \lambda_{1}(s) d s+E \int_{0}^{t \wedge \rho_{k}}\left[\mu_{2}\left(x\left(s^{-}-\tau\left(s^{-}\right)\right)\right)-\mu_{1}\left(x\left(s^{-}\right)\right)\right] d s \\
& \leq V(0, \xi)+\int_{0}^{t} \lambda_{1}(s) d s+E \int_{-\tau}^{0} \mu_{2}(x(\theta)) d \theta+E \int_{0}^{t \wedge \rho_{k}}\left[\mu_{2}\left(x\left(s^{-}\right)-\mu_{1}\left(x\left(s^{-}\right)\right)\right] d s\right. \\
& \leq V(0, \xi)+\int_{0}^{t} \lambda_{1}(s) d s+E \int_{-\tau}^{0} \mu_{2}(x(\theta)) d \theta
\end{aligned}
$$


Moreover, we have

$$
P\left(\rho_{k} \leq t\right) \leq \frac{V(0, \xi)+\int_{0}^{t} \lambda_{1}(s) d s+E \int_{-\tau}^{0} \mu_{2}(x(\theta)) d \theta}{\inf _{|x| \geq k, t \geq 0} V(t, x(t))}
$$

It's clear that $P\left(\rho_{e} \leq t\right)=0$ with letting $k \rightarrow \infty$ and (3.1), due to the arbitrary of $t$, so we obtain $\rho_{e}=\infty$, a.s..

Definition 3.1. The system (2.6) is said to be almost surely asymptotically stable if for any $x_{0}=$ $\xi \in R^{n}, \lim _{t \rightarrow \infty} x(t, \xi)=0$, a.s..

In the rest of this section, the main results have been presented, let us give the following theorem,

Theorem 3.1. Let Assumption II and III hold, and moreover, suppose that the function $V \in$ $C^{1,2}\left(R_{+} \times R^{n} ; R_{+}\right)$in assumption III satisfies, for all $x \in R^{n}, t \geq 0$,

$$
\alpha_{1}(t,|x|) \leq V(t, x) \leq \alpha_{2}(t,|x|) .
$$

where $\alpha_{1}(t, x), \alpha_{2}(t, x)$ belong to $\mathcal{K}_{\infty}$ with respect to $x$. Then for any initial data $\xi$, the global solution $x(t ; \xi)$ of system $(2.6)$ is almost surely asymptotically stable, i.e.

$$
\lim _{t \rightarrow \infty} x(t ; \xi)=0, a . s .
$$

Proof. Due to the conditions of Lemma 3.1 hold, for any $\xi \in R^{n}$, the system (2.6) admits a global solution $x(t, \xi)$, for the simplicity, we will write the $x(t)$ instead of $x(t, \xi)$. It is clear that this theorem holds because of the solution $x(t) \equiv 0$ a.s. for $\xi=0$. So for $\xi \neq 0$, we have the following proof. Due of the complexity of proof, so we divide the proof into three steps as follows.

Step 1: In this step, we will show that the system (2.6) is stable in probability and the sample space is divided, applying the Itô formula to $V(t, x)$, and then using the Assumption III, for any 
$t>0$, we have

$$
\begin{aligned}
V(t, x(t))= & V(0, \xi)+\int_{0}^{t} L V\left(s, x\left(s^{-}\right), x\left(s^{-}-\tau\left(s^{-}\right)\right)\right) d s+\sum_{j=1}^{p} \int_{0}^{t} V_{x}\left(s, x\left(s^{-}\right)\right) K_{j}^{*} x\left(s^{-}\right) d B_{j}(s) \\
& +\sum_{i=1}^{m} \int_{0}^{t} \int_{R^{m} \backslash\{0\}}\left[V\left(s, x\left(s^{-}\right)+K_{i} x\left(s^{-}\right) y_{i}\right)-V\left(s, x\left(s^{-}\right)\right)\right] \tilde{N}(d s, d y) \\
\leq & V(0, \xi)+\int_{0}^{t} \lambda(s) d s+\int_{0}^{t}\left(\mu_{2}\left(x\left(s^{-}-\tau\left(s^{-}\right)\right)\right)-\mu_{1}\left(x\left(s^{-}\right)\right)\right) d s \\
& +\sum_{j=1}^{p} \int_{0}^{t} V_{x}\left(s, x\left(s^{-}\right)\right) K_{j}^{*} x\left(s^{-}\right) d B_{j}(s) \\
& +\sum_{i=1}^{m} \int_{0}^{t} \int_{R^{m} \backslash\{0\}}\left[V\left(s, x\left(s^{-}\right)+K_{i} x\left(s^{-}\right) y_{i}\right)-V\left(s, x\left(s^{-}\right)\right)\right] \tilde{N}(d s, d y) \\
\leq & V(0, \xi)+\int_{0}^{t} \lambda(s) d s+\int_{-\tau}^{0} \mu_{2}(x(\theta)) d \theta+\sum_{j=1}^{p} \int_{0}^{t} V_{x}\left(s, x\left(s^{-}\right)\right) K_{j}^{*} x\left(s^{-}\right) d B_{j}(s) \\
& +\sum_{i=1}^{m} \int_{0}^{t} \int_{R^{m} \backslash\{0\}}\left[V\left(s, x\left(s^{-}\right)+K_{i} x\left(s^{-}\right) y_{i}\right)-V\left(s, x\left(s^{-}\right)\right)\right] \tilde{N}(d s, d y) \\
\doteq & V_{t}(\xi)+M(t)
\end{aligned}
$$

where $V_{t}(\xi)=V(0, \xi)+\int_{0}^{t} \lambda(s) d s+\int_{-\tau}^{0} \mu_{2}(x(\theta)) d \theta$ is bounded, because of $\xi \in D_{\mathcal{F}_{0}}^{b}\left([-\tau, 0] ; R^{n}\right)$ and $\int_{0}^{\infty} \lambda(t) d t<\infty, M(t)$ represents the rest part of above the equation, which yields that $V(t, x(t)), t \geq$ 0 is a supermartingale with respect to the filtration $\left\{\mathcal{F}_{t}\right\}_{t \geq 0}$ generated by $B(\cdot)$ and $\tilde{N}(\cdot, \cdot)$. Using the supermartingale inequality (Rogers\&Williams[22],p154,(54.5)), for any function $\delta(\cdot) \in \mathcal{K}_{\infty}$, yield

$$
P\left\{\sup _{0 \leq s \leq t} V(s, x(s)) \geq \delta\left(V_{t}(\xi)\right)\right\} \leq \frac{21 V_{t}(\xi)}{\delta\left(V_{t}(\xi)\right)}, t \geq 0,
$$

in other word,

$$
P\left\{\sup _{0 \leq s \leq t} V(s, x(s))<\delta\left(V_{t}(\xi)\right)\right\} \geq 1-\frac{21 V_{t}(\xi)}{\delta\left(V_{t}(\xi)\right)}, t \geq 0 .
$$

Note that $\sup _{0 \leq s \leq t} V(s, x(s))<\delta\left(V_{t}(\xi)\right) \operatorname{implies} \sup _{0 \leq s \leq t}|x|<v_{t}\left(V_{t}(\xi)\right)$, where $v_{t}=\alpha_{1}^{-1} \circ \delta$, and $\alpha_{1}^{-1}$ is the inverse function of $\alpha_{1}$ with respect to $x$. For (3.3) and given $\epsilon>0$, we can obtain the $\frac{21 V_{t}(\xi)}{\delta\left(V_{t}(\xi)\right)} \leq \epsilon$ with choosing appropriate $\delta(\cdot)$, then for $t>0$,

$$
P\left\{\sup _{0 \leq s \leq t}|x(s)|<v_{t}\left(V_{t}(\xi)\right)\right\} \geq 1-\epsilon, t \geq 0 .
$$

This yields

$$
P\left\{|x(s)|<v_{t}\left(V_{t}(\xi)\right)\right\} \geq 1-\epsilon, t \geq 0 .
$$

Let us decompose the sample space

$$
\begin{aligned}
& \Omega_{1}=\left\{\omega: \limsup _{t \rightarrow \infty} \mu(x(t, \omega))=0\right\} \\
& \Omega_{2}=\left\{\omega: \liminf _{t \rightarrow \infty} \mu(x(t, \omega))>0\right\}
\end{aligned}
$$




$$
\Omega_{3}=\left\{\omega: \liminf _{t \rightarrow \infty} \mu(x(t, \omega))=0 \text { and } \limsup _{t \rightarrow \infty} \mu(x(t, \omega))>0\right\},
$$

in order to obtain the results, we will show that $P\left(\Omega_{2}\right)=P\left(\Omega_{3}\right)=0$, which imply that $P\left(\Omega_{1}\right)=1$.

Step 2: In this step, we will show that $P\left(\Omega_{2}\right)=0$, using the Itô formula and Assumption III, we have

$$
\begin{aligned}
E V(t, x(t)) & =V(0, \xi)+E\left\{\int_{0}^{t} L V\left(s, x\left(s^{-}\right), x\left(s^{-}-\tau\left(s^{-}\right)\right)\right) d s\right\} \\
& \leq V_{t}(\xi)-E\left\{\int_{0}^{t} \mu(x(s)) d s\right\}
\end{aligned}
$$

Since $V(t, x) \geq 0$, this yields $E\left\{\int_{0}^{t} \mu(x(s)) d s\right\} \leq V_{t}(\xi)$, letting $t \rightarrow \infty$, and using the fatou's lemma, then $E\left\{\int_{0}^{\infty} \mu(x(s)) d s\right\} \leq C_{V_{t}}$, where $C_{V_{t}}$ is the upper bounded of $V_{t}(\xi)$, and due to the nonnegative function $\mu$, hence $\int_{0}^{\infty} \mu(x(s)) d s \leq C_{V_{t}}$, which implies that $P\left(\Omega_{2}\right)=0$.

Step 3: In this step, we will show that $P\left(\Omega_{3}\right)=0$, and proceed by contradiction, if it is not true, then there exist $\epsilon_{0}>0$ and $\epsilon_{1}>0$, such that

$$
P\left\{\mu(x(\cdot)) \text { cross from below } \epsilon_{1} \text { to above } 2 \epsilon_{1} \text { and back infinitely many times }\right\} \geq \epsilon_{0} .
$$

Now recalling the definition of $\rho_{k}$ and the boundedness of the initial data, combining the Assumption II, there exists a constant $C_{k}>0$ such that $\sup _{t \geq 0}\left|f\left(t \wedge \rho_{k}, x\left(t \wedge \rho_{k}\right), x\left(t \wedge \rho_{k}-\tau\left(t \wedge \rho_{k}\right)\right)\right)\right| \leq C_{k}$. Computing

$$
\begin{aligned}
E & \left\{\sup _{0 \leq s \leq t}\left|x\left(t \wedge \rho_{k}\right)-\xi\right|^{2}\right\} \\
= & E\left\{\sup _{0 \leq s \leq t} \mid \int_{0}^{s \wedge \rho_{k}}\left(f\left(h, x\left(h^{-}\right), x\left(h^{-}-\tau\left(h^{-}\right)\right)\right)+\sum_{i=1}^{m} K_{i} x\left(h^{-}\right) \tilde{b}_{i}\right) d h\right. \\
& \left.+\sum_{j=1}^{p} \int_{0}^{s \wedge \rho_{k}} K_{j}^{*} x\left(h^{-}\right) d B_{j}(h)+\left.\sum_{i=1}^{m} \int_{0}^{s \wedge \rho_{k}} \int_{R^{m} \backslash\{0\}} K_{i} x\left(h^{-}\right) y_{i} \tilde{N}(d h, d y)\right|^{2}\right\} \\
\leq & 3 E\left\{\sup _{0 \leq s \leq t}\left|\int_{0}^{s \wedge \rho_{k}}\left(f\left(h, x\left(h^{-}\right), x\left(h^{-}-\tau\left(h^{-}\right)\right)\right)+\sum_{i=1}^{m} K_{i} x\left(h^{-}\right) \tilde{b}_{i}\right) d h\right|^{2}\right\} \\
& +3 E\left\{\sup _{0 \leq s \leq t}\left|\sum_{j=1}^{p} \int_{0}^{s \wedge \rho_{k}} K_{j}^{*} x\left(h^{-}\right) d B_{j}(h)\right|^{2}\right\} \\
& +3 E\left\{\sup _{0 \leq s \leq t}\left|\sum_{i=1}^{m} \int_{0}^{s \wedge \rho_{k}} \int_{R^{m} \backslash\{0\}} K_{i} x\left(h^{-}\right) y_{i} \tilde{N}(d h, d y)\right|^{2}\right\} \\
\leq & 3 C_{k, K_{i}, \tilde{b}_{i}}^{2} t^{2}+3 E\left\{\sup _{0 \leq s \leq t}\left|\sum_{j=1}^{p} \int_{0}^{s \wedge \rho_{k}} K_{j}^{*} x\left(h^{-}\right) d B_{j}(h)\right|^{2}\right\} \\
& +3 E\left\{\sup _{0 \leq s \leq t}\left|\sum_{i=1}^{m} \int_{0}^{s \wedge \rho_{k}} \int_{R^{m} \backslash\{0\}} K_{i} x\left(h^{-}\right) y_{i} \tilde{N}(d h, d y)\right|^{2}\right\}
\end{aligned}
$$

Combining the Burkholder's inequality (Applebaum[1], Chap 4,Theorem 4.4.21) and (Applebaum[1], Chap 
4,Theorem 4.4.22), Doob's martingale inquality, we obtain

$$
\begin{aligned}
& E\left\{\sup _{0 \leq s \leq t}\left|\sum_{j=1}^{p} \int_{0}^{s \wedge \rho_{k}} K_{j}^{*} x\left(h^{-}\right) d B_{j}(h)\right|^{2}\right\} \\
& \leq 4 E\left\{\sum_{j=1}^{p} \int_{0}^{t \wedge \rho_{k}}\left|K_{j}^{*} x\left(h^{-}\right)\right|^{2} d h\right\} \\
& \leq 4 C_{k, K_{j}^{*}}^{2} t
\end{aligned}
$$

Applying the Kunitas first inequality inequality (Applebaum[1],Chap 4,Theorem 4.4.23), we get

$$
\begin{aligned}
& E\left\{\sup _{0 \leq s \leq t}\left|\sum_{i=1}^{m} \int_{0}^{s \wedge \rho_{k}} \int_{R^{m} \backslash\{0\}} K_{i} x\left(h^{-}\right) y_{i} \tilde{N}(d h, d y)\right|^{2}\right\} \\
& \leq C E\left\{\sum_{i=1}^{m} \int_{0}^{t \wedge \rho_{k}} \int_{R^{m} \backslash\{0\}}\left|K_{i} x\left(h^{-}\right) y_{i}\right|^{2} \pi(d y) d h\right\} \\
& \leq C_{k, K_{i}}^{2} t
\end{aligned}
$$

Substituting (3.9) and (3.10) into (3.8), this yields

$$
E\left\{\sup _{0 \leq s \leq t}\left|x\left(s \wedge \rho_{k}\right)-\xi\right|^{2}\right\} \leq 3 C_{k, K_{i}, \tilde{b}_{i}}^{2} t^{2}+12 C_{k, K_{j}^{*}}^{2} t+3 C_{k, K_{i}}^{2} t
$$

and by the chebyshev's inequality, for any $\vartheta>0$,

$$
\begin{aligned}
& P\left\{\sup _{0 \leq s \leq t}\left|x\left(s \wedge \rho_{k}\right)-\xi\right|>\vartheta\right\} \\
& \leq \frac{E\left\{\sup _{0 \leq s \leq t}\left|x\left(s \wedge \rho_{k}\right)-\xi\right|^{2}\right\}}{\vartheta^{2}} \\
& \leq \frac{3 C_{k, K_{i}, \tilde{b}_{i}}^{2} t^{2}+12 C_{k, K_{j}^{*}}^{2} t+3 C_{k, K_{i}}^{2} t}{\vartheta^{2}}
\end{aligned}
$$

Since $\mu(\cdot)$ is continuous, it must be uniformly continuous in the closed ball $\mathcal{O}:=\left\{x \in R^{n}:|x| \leq\right.$ $\left.v_{t}(k)\right\}$, where $v_{t}=\alpha_{1}^{-1} \circ \delta$. For given $u>0$, a function $\gamma \in \mathcal{K}$ have been chosen, which such that for any $x, y \in \mathcal{O},|x-y| \leq \gamma(u)$, implies $|\mu(x)-\mu(y)| \leq u$. Then, for $|\xi| \leq k$ and $\epsilon_{2}>0$,

$$
\begin{aligned}
& P\left\{\sup _{0 \leq s \leq t}|\mu(x(s))-\mu(\xi)|>\epsilon_{2}\right\} \\
& \leq P\left\{\sup _{0 \leq s \leq t}|x(s)-\xi|>\gamma\left(\epsilon_{2}\right) \text { and } \sup _{0 \leq s \leq t}|x(s)|<v_{t}(k)\right\}+P\left\{\sup _{0 \leq s \leq t}|x(s)| \geq v_{t}(k)\right\} \\
& \leq P\left\{\sup _{0 \leq s \leq t}\left|x\left(s \wedge \rho_{v(t, k)}\right)-\xi\right|>\gamma\left(\epsilon_{2}\right)\right\}+P\left\{\sup _{0 \leq s \leq t}|x(s)| \geq v_{t}(k)\right\} \\
& \leq \frac{3 C_{k, K_{i}, \tilde{b}_{i}}^{2} t^{2}+12 C_{k, K_{j}^{*}}^{2} t+3 C_{k, K_{i}}^{2} t}{\gamma\left(\epsilon_{2}\right)^{2}}+\epsilon
\end{aligned}
$$

Setting $\epsilon=\frac{1}{2}$, for any $\epsilon_{2}>0$, there exists $t^{*}=t^{*}\left(k, \epsilon_{2}\right)$ such that

$$
P\left\{\sup _{0 \leq s \leq t}|\mu(x(s))-\mu(\xi)| \leq \epsilon_{2}\right\} \geq \frac{1}{4}, \quad \forall t \in\left(0, t^{*}\right] .
$$


Step 3: Let us define a sequence of stopping times,

$$
\begin{gathered}
\mathcal{T}_{1}:=\inf \left\{t \geq 0: \mu(x(t))<\epsilon_{1}\right\}, \\
\mathcal{T}_{2 n}:=\inf \left\{t \geq \mathcal{T}_{2 n-1}: \mu(x(t))>2 \epsilon_{1}\right\}, \quad n=1,2, \ldots, \\
\mathcal{T}_{2 n+1}:=\inf \left\{t \geq \mathcal{T}_{2 n}: \mu(x(t))<\epsilon_{1}\right\}, \quad n=1,2, \ldots,
\end{gathered}
$$

and we set $\inf \emptyset=\infty$. By (3.6), it is easy to get

$$
\begin{aligned}
\infty & >E \int_{0}^{\infty} \mu(x(s)) d s \\
& \left.\geq \sum_{n=1}^{\infty} E\left[I_{\{} \mathcal{T}_{2 n}<\rho_{k}\right\} \int_{\mathcal{T}_{2 n}}^{\mathcal{T}_{2 n+1}} \mu(x(s)) d s\right] \\
& \left.\geq \epsilon_{1} \sum_{n=1}^{\infty} E\left[I_{\{} \mathcal{T}_{2 n}<\rho_{k}\right\}\left(\mathcal{T}_{2 n+1}-\mathcal{T}_{2 n}\right)\right] \\
& \left.=\epsilon_{1} \sum_{n=1}^{\infty} E\left[I_{\{} \mathcal{T}_{2 n}<\rho_{k}\right\} E\left(\mathcal{T}_{2 n+1}-\mathcal{T}_{2 n} \mid \mathcal{F}_{\mathcal{T}_{2 n}}\right)\right]
\end{aligned}
$$

By the strong Markov property of solution $x(t)$ that on $\left\{\mathcal{T}_{2 n}<\rho_{k}\right\}$, and setting $\epsilon_{1}=2 \epsilon_{2}$, we obtain

$$
\begin{aligned}
& E\left(\mathcal{T}_{2 n+1}-\mathcal{T}_{2 n} \mid \mathcal{F}_{\mathcal{T}_{2 n}}\right) \\
& \left.\geq E\left[\left(\mathcal{T}_{2 n+1}-\mathcal{T}_{2 n}\right) I_{\{} \sup _{0 \leq s \leq t^{*}}|\mu(\tilde{x}(s))-\mu(\tilde{\xi})| \leq \frac{\epsilon_{1}}{2}\right\} \mid \mathcal{F}_{\mathcal{T}_{2 n}}\right] \\
& \geq t^{*} P\left\{\sup _{0 \leq s \leq t^{*}}|\mu(\tilde{x}(s))-\mu(\tilde{\xi})| \leq \frac{\epsilon_{1}}{2} \mid \mathcal{F}_{\mathcal{T}_{2 n}}\right\} \\
& \geq \frac{t^{*}}{4}
\end{aligned}
$$

where $t^{*}=t^{*}\left(k, \epsilon_{1} / 2\right)$ and $\tilde{x}=x\left(\cdot+\mathcal{T}_{2 n}\right)$, we substitute (3.16) into (3.15), and have

$$
\frac{t^{*} \epsilon_{1}}{4} \sum_{n=1}^{\infty} P\left\{\mathcal{T}_{2 n}<\rho_{k}\right\}<\infty,
$$

applying the Borel-Cantelli lemma, this yields

$$
P\left\{\mathcal{T}_{2 n}<\rho_{k} \text { for infinitely many } n\right\}=0 .
$$

Since

$\left\{\mathcal{T}_{2 n}<\rho_{k}\right.$ for infinitely many $\left.n\right\}=\left\{\mathcal{T}_{2 n}<\rho_{k}\right.$ for infinitely many $n$ and $\left.\rho_{k}=\infty\right\}$

$$
\bigcup\left\{\mathcal{T}_{2 n}<\rho_{k} \text { for infinitely many } n \text { and } \rho_{k}<\infty\right\} \text {, }
$$

then

$$
P\left\{\mathcal{T}_{2 n}<\infty \text { for infinitely many } n \text { and } \rho_{k}=\infty\right\}=0 .
$$

By the (3.2), for any $k>0$,

$$
\begin{aligned}
P\left\{\rho_{k}=\infty\right\} & \geq P\left\{\sup _{t \geq 0}|x(t)|<k\right\} \geq P\left\{\sup _{t \geq 0} \mid V\left(t,(x(t)) \mid<\alpha_{1}(t, k)\right\}\right. \\
& \geq 1-\frac{V(0, \xi)}{\alpha_{1}(t, k)}
\end{aligned}
$$


and then letting $k \rightarrow \infty$, we obtain $P\left\{\rho_{k}=\infty\right\} \rightarrow 1$, combining with (3.18), this yields

$$
P\left\{\mathcal{T}_{2 n}<\infty \text { for infinitely many } n\right\}=0,
$$

which is the contradiction with (3.7), hence $P\left(\Omega_{3}\right)=0$, we can implies that $\lim _{t \rightarrow \infty} \mu(x(t))=0$ a.s., together with the property of the function $\mu(0)=0$, yields $\lim _{t \rightarrow \infty} x(t)=0$ a.s., the proof is completed.

Before the end of this section, we present the following example, which is one-dimension case and illustrates Theorem 3.1.

Example 3.1. Consider the scalar stochastic differential equations with jumps in the form

$$
\begin{aligned}
& d x(t)=\left[K \tilde{b}-x^{2}\left(t^{-}\right)\right] x\left(t^{-}\right) d t+\sigma K x\left(t^{-}\right) d B(t)+\int_{0}^{\infty} K x\left(t^{-}\right) y \tilde{N}(d t, d y), t>0 \\
& x(0)=x_{0},
\end{aligned}
$$

where $K, \tilde{b}, \sigma \in R$ are constants, $B(t)$ is a scalar standard Brownian motion, and $\tilde{N}(\cdot, \cdot)$ is a compensated Poisson random measure.

Let $V(t, x)=x^{2}$ for any $x \in R$, we obtain

$$
L V(t, x) \leq\left(2 K \tilde{b}+\sigma^{2} K^{2}+2 K^{2} \int_{0}^{1} y^{2} \pi(d y)+2 K \int_{1}^{\infty} y \pi(d y)\right) x^{2},
$$

then, by Theorem 3.1, the solution of system (3.21) is almost surely asymptotically stable with choosing the appropriate constant $K$ in the open interval $\left(-\left(\tilde{b}+\int_{1}^{\infty} y \pi(d y)\right) /\left(\sigma^{2} / 2+\int_{0}^{1} y^{2} \pi(d y)\right), 0\right)$ as the feedback control part, such that

$$
2 K \tilde{b}+\sigma^{2} K^{2}+2 K^{2} \int_{0}^{1} y^{2} \pi(d y)+2 K \int_{1}^{\infty} y \pi(d y)<0 .
$$

\section{$4 \quad$ LMIs approach analysis}

Now, let us discuss the design of controller $u(x(t))=\left(K_{1} x(t), K_{2} x(t), \ldots, K_{m} x(t)\right)$, the following theorem describes a method to find the matrix $K_{i}$ by solving a set of linear matrix inequalities(LMIs). In order to obtain the following theorem, we present the necessary assumption and lemma.

Assumption IVAssume that

$$
|f(t, x, \bar{x})|^{2} \leq x^{T} F_{1} x+\bar{x} F_{2} \bar{x},
$$

where $F_{1}, F_{2} \in R^{n \times n}$ are positive definite matrices.

Applying the Assumption I, for any positive definite matrix $P=P^{T}>0$, we have

$$
\begin{aligned}
& \sum_{i=1}^{m} \int_{R^{m} \backslash\{0\}}\left[\left(K_{i} x y_{i}\right)^{T} P\left(K_{i} x y_{i}\right)+\left(K_{i} x y_{i}\right)^{T} P x-x^{T} P K_{i} x y_{i}\right] \pi(d y) \\
& \leq C_{r} \sum_{i=1}^{m}\left[\left(K_{i} x\right)^{T} P\left(K_{i} x\right)+\left(K_{i} x\right)^{T} P x-x^{T} P K_{i} x\right],
\end{aligned}
$$

where $C_{r}$ is constant, which depend on the Assumption I. 
Lemma 4.1. (Xu[26])For any constant matrix $M \in R^{n \times m}$, inequality

$$
2 u^{T} M v \leq \varepsilon u^{T} M G M^{T} u+\frac{1}{\varepsilon} v^{T} G^{-1} v, u \in R^{n}, v \in R^{m}
$$

holds for any pair of symmetric positive definite matrix $G \in R^{m \times m}$ and number $\varepsilon>0$.

Theorem 4.1. Let Assumption IV holds, and if there exist the constants $\varepsilon>0, \delta>0$, and positive definite matrix $P=P^{T}>0$, matrices $Y_{i}(i=1,2, \ldots, m)$, such that the following LMIs

$$
\Xi=\left[\begin{array}{cccc}
\Phi_{1} & \Phi_{2} & \Phi_{4} & \Phi_{6} \\
* & -\Phi_{3} & 0 & 0 \\
* & * & -\Phi_{5} & 0 \\
* & * & * & -\Phi_{7}
\end{array}\right]<0
$$

hold, where

$$
\begin{gathered}
\Phi_{1}=\varepsilon I+\sum_{i=1}^{m} \tilde{b}_{i} Y_{i}+\sum_{i=1}^{m} \tilde{b}_{i} Y_{i}^{T}-\sum_{i=1}^{m} C_{r} Y_{i}+\sum_{i=1}^{m} C_{r} Y_{i}^{T}, \\
\Phi_{2}=\operatorname{diag}\left(\varepsilon F_{1}^{-1},\left(1-d_{\tau}\right) \varepsilon F_{2}^{-1}\right), \\
\Phi_{3}=(X, X), \\
\Phi_{4}=\left(\sqrt{\sum_{j=1}^{p} \sigma_{1 j}^{2} Y_{1}}, \sqrt{\sum_{j=1}^{p} \sigma_{2 j}^{2} Y_{2}}, \ldots, \sqrt{\sum_{j=1}^{p} \sigma_{m j}^{2}} Y_{m}\right) \\
\Phi_{5}=\operatorname{diag}\left((\delta(m-1)+1) X,\left(\delta(m-2)+\frac{1}{\delta}+1\right) X, \ldots,\left(\frac{m-1}{\delta}+1\right) X\right), \\
\Phi_{6}=\left(Y_{1}, Y_{2}, \ldots, Y_{m}\right), \\
\Phi_{7}=\operatorname{diag}\left(\frac{1}{C_{r}} X, \frac{1}{C_{r}} X, \ldots, \frac{1}{C_{r}} X\right) .
\end{gathered}
$$

Then the controlled system (2.6) is almost surely asymptotically stable, and the feedback controller is designed as follows,

$$
u(x(t))=\left(K_{1} x(t), K_{2} x(t), \ldots, K_{m} x(t)\right), K_{i}=Y_{i} X^{-1}, i=1,2, \ldots, m .
$$

Proof. Let $P=X^{-1}$ and $V(x)=x^{T}(t) P x(t)+\int_{t-\tau(t)}^{t} x^{T}(s) Q x(s) d s$, using the Assumption IV 
and lemma 4.1, we obtain

$$
\begin{aligned}
L V(t, x, \bar{x})= & x^{T} Q x-(1-\tau(t)) \bar{x}^{T} Q \bar{x}+2 x^{T} P\left[f(t, x, \bar{x})+\sum_{i=1}^{m} K_{i} x \tilde{b}_{i}\right]+\sum_{j=1}^{p}\left[\left(K_{j}^{*} x\right)^{T} P\left(K_{j}^{*} x\right)\right] \\
& +\sum_{i=1}^{m} \int_{R^{m} \backslash\{0\}}\left[\left(x+K_{i} x y_{i}\right)^{T} P\left(x+K_{i} x y_{i}\right)-x^{T} P x-2 x^{T} P K_{i} x y_{i}\right] \pi(d y) \\
\leq & x^{T}\left\{Q+\varepsilon P^{2}+\frac{1}{\varepsilon} F_{1}+\sum_{i=1}^{m} P K_{i} \tilde{b}_{i}+\sum_{i=1}^{m}\left(K_{i} \tilde{b}_{i}\right)^{T} P\right. \\
& +\sum_{i=1}^{m}\left[\left(\delta(m-i)+\frac{i-1}{\delta}+1\right)\left(\sum_{j=1}^{p} \sigma_{i j}^{2}\right) K_{i}^{T} P K_{i}\right]+C_{r}\left[\sum_{i=1}^{m} K_{i}^{T} P K_{i}\right] \\
& \left.-C_{r} \sum_{i=1}^{m} P K_{i}+C_{r} \sum_{i=1}^{m} K_{i}^{T} P\right\} x+\bar{x}^{T}\left[\frac{1}{\varepsilon} F_{2}-\left(1-d_{\tau}\right) Q\right] \bar{x} \\
\doteq & -x^{T} \Theta_{1} x+\left(1-d_{\tau}\right) \bar{x}^{T} \Theta_{2} \bar{x},
\end{aligned}
$$

where

$$
\begin{aligned}
\Theta_{1} & =-Q-\varepsilon P^{2}-\frac{1}{\varepsilon} F_{1}-\sum_{i=1}^{m} P K_{i} \tilde{b}_{i}-\sum_{i=1}^{m}\left(K_{i} \tilde{b}_{i}\right)^{T} P-\sum_{i=1}^{m}\left[\left(\delta(m-i)+\frac{i-1}{\delta}+1\right)\left(\sum_{j=1}^{p} \sigma_{i j}^{2}\right) K_{i}^{T} P K_{i}\right] \\
& -C_{r}\left[\sum_{i=1}^{m} K_{i}^{T} P K_{i}\right]+C_{r} P K_{i}-C_{r} K_{i}^{T} P \\
\Theta_{2} & =\frac{1}{\varepsilon\left(1-d_{\tau}\right)} F_{2}-Q .
\end{aligned}
$$

Viewing that $P=X^{-1}$ and $K_{i}=Y_{i} X^{-1}$, we pre-multiply and post-multiply (4.1) by the matrix $\operatorname{diag}(P, P, \ldots, P)$, then we can have the following inequality with using Schur complements lemma,

$$
\begin{aligned}
& \varepsilon P^{2}+\frac{1}{\varepsilon} F_{1}+\sum_{i=1}^{m} P K_{i} \tilde{b}_{i}+\sum_{i=1}^{m}\left(K_{i} \tilde{b}_{i}\right)^{T} P+\sum_{i=1}^{m}\left[\left(\delta(m-i)+\frac{i-1}{\delta}+1\right)\left(\sum_{j=1}^{p} \sigma_{i j}^{2}\right) K_{i}^{T} P K_{i}\right] \\
& +C_{r}\left[\sum_{i=1}^{m} K_{i}^{T} P K_{i}\right]-C_{r} P K_{i}+C_{r} K_{i}^{T} P+\frac{1}{\varepsilon\left(1-d_{\tau}\right)} F_{2}<0
\end{aligned}
$$

yield $-\Theta_{1}+\Theta_{2}<0$, which implies $\Theta_{1}>\Theta_{2}$, and let $\mu_{1}(x)=x^{T} \Theta_{1} x, \mu_{2}(x)=\left(1-d_{\tau}\right) x^{T} \Theta_{2} x$, the conditions of Theorem 3.1 have been satisfied, so the controlled system (2.6) is almost surely asymptotically stable, and the proof is completed.

\section{Discussion of main resuts}

The Lévy process is employed to stabilize the system, which generalize the Brownian motion as the noise. In the main results of this paper, we employ more stopping time theory to obtain the 
almost sure asymptotic sense stabilization with usual conditions of stability, comparing with some references, almost sure asymptotic sense is more general and more stochastic techniques appear in the rigorous proof. Meanwhile, the delay mode-dependent case and functional equation case can be studied with the similar technique. The diffusion part of system (2.2) is a bit simple, in the subsequent research, we will present the complex design, which proposes the new challenge after Lévy-Itô decomposition of Lévy process, especially the design of the cotroller with LMIs approach. On the other hand, as the opposite aspect, destabilization arises our interest, the more important application is the biological systems, the animals population always change from the stable status to unstable one, then go to another stable one, which is variation for adapting to the environment, so the unstable status is also important, which will be also in the subsequent research.

\section{Acknowledgment}

This work was partially supported by PNSF of Anhui(1208085QG131,KJ2013Z008), SRFDP of China (20133219110040), NNSF of China(11301001), HSSF for the Ministry of Education(10YJC630143), EYSF of Anhui Province of China (2013SQRL030ZD).

\section{References}

[1] Applebaum D.. Lévy Processes and Stochastic Calculus, second edition. Cambridge University Press, 2009.

[2] Applebaum D.,Siakalli M.. Asymptotic stability of stochastic diffrential equations driven by Lévy noise. Journal of Applied Probability 46, (2009),1116-1129.

[3] Appleby J., Mao X., and Rodkina A.. Stabilization and Destabilization of Nonlinear Differential Equations by Noise. IEEE Transactiions on autimatic control, 53(3), (2008),683-691.

[4] Arnold L., Crauel H., Wihstutz V.. Stabilization of linear systems by noise. SIAM Journal on Control and Optimization, 21, (1983),451-461.

[5] Bao J., Yuan C.. Stabilization of partial differential equations by Lévy noise. Stoch. Anal. Appl., 30(2), (2012), 354-374.

[6] Bao J., Yuan C.. Long-term behavior of stochastic interest rate models with jumps and memory. Insurance Math. Econom., 53(1), (2013), 266-272.

[7] Bellman R., Bentsman J., Meerkov S.. Stability for fast periodic systems. IEEE Transactions on Automatic Control, AC-30,(1985), 289-291.

[8] Bercu B., Dufour F., and Yin G.. Almost sure stabilization for feedback controls of regimeswitching linear systems with a hidden Markov Chain. IEEE Transactions on Automatic Control, 54(9), (2009), 2114-2125.

[9] Deng H., Krstic M. and Williams J..Stabilization of Stochastic Nonlinear Systems Driven by Noise of Unknown Covariance.IEEE Transactions on Automatic Control, 46(8),(2001), 12371253 . 
[10] Hu L., Mao X.. Almost sure exponential stabilisation of stochastic systems by state-feedback control. Automatica, 44(2),(2008),465-471.

[11] Hu J., Wang Z., Gao H., and Stergioulas L. K.. Robust sliding mode control for discrete stochastic systems with mixed time delays, randomly occurring uncertainties, and randomly occurring nonlinearities. IEEE Transactions on Industrial Electronics, 59(7),(2012),3008-3015.

[12] Huang L.. Stochastic stabilization and destabilization of nonlinear differential equations.Systems and Control Letters, 62,(2013),163-169.

[13] Huang L., Mao X.. On Almost Sure Stability of Hybrid Stochastic Systems With ModeDependent Interval Delays.IEEE Transactiions on autimatic control, 55(8),(2010), 1946-1952.

[14] Khasminski R. Z.. Stochastic Stability of Differential Equations, Alphen: Sojtjo? and Noordhoff(1980), translation of the Russian edition (1969).

[15] Li X. and De Souza C. E.. Delay-dependent robust stability and stabilization of uncertain linear delay systems: a linear matrix inequality approach. IEEE Transactions on Automatic Control, 42(8),(1997), 1144-1148.

[16] Liu, D., Wang, W., Ignatyev, O., Zhang, W.. Partial stochastic asymptotic stability of neutral stochastic functional differential equations with Markovian switching by boundary condition. Adv. Difference Equ. 220(2012).

[17] Liu L., Shen Y., and Jiang F.. The almost sure asymptotic stability and th moment asymptotic stability of nonlinear stochastic differential systems with polynomial growth. IEEE Transactions on Automatic Control, 56(8),(2011), 1985-1990.

[18] Mao X.. Stochastic versions of the LaSalle theorem. Journal of Differential Equations, 153(1),(1999) 175-195.

[19] Mao, X.. Stochastic differential equations and application. Horwood Publications, Chichester (2007).

[20] Mao X., Shen Y., and Yuan C.. Almost surely asymptotic stability of neutral stochastic differential delay equations with Markovian switching. Stochastic Processes and their Applications, 118(8),(2008), 1385-1406.

[21] Mao X., Lam J., and Huang L.. Stabilisation of hybrid stochastic differential equations by delay feedback control. Systems and Control Letters, 57(11), (2008),927-935.

[22] Rogers L. C. G., and Williams D.. Diffusions, Markov Processes and Martingales, 2nd ed. Cambridge University Press, 2000, vol. 1.

[23] Shen B., Wang Z., Hung Y., and Chesi G.. Distributed H filtering for polynomial nonlinear stochastic systems in sensor networks. IEEE Transactions on Industrial Electronics, 58(5), (2011), 1971-1979.

[24] Wei G., Wang Z., and Shu H.. Nonlinear H control of stochastic time-delay systems with Markovian switching. Chaos, Solitons and Fractals, 35(3), (2008), 442-451.

[25] Xie Q., Han Z., Wang H. and Zhang W..Stabilization of center systems via immersion and invariance. Nonlinear Dynamics, 57(3), (2009), 313-319. 
[26] Xu B.. Stability robustness bounds for linear systems with multiple time-varying delayed perturbations. Int. J. Syst. Sci., 28,(1997), 1311-1317.

[27] Yang H., Shu H., Kan X., and Che Y..Almost Sure Stability and Stabilization for Hybrid Stochastic Systems with Time-Varying Delays. Hindawi Publishing Corporation Mathematical Problems in Engineering, Volume 2012,Article ID 183729.

[28] Yuan C. and Mao X.. Robust stability and controllability of stochastic differential delay equations with Markovian switching. Automatica, 40(3), (2004),343-354.

[29] Yue D. and Han Q.. Delay-dependent exponential stability of stochastic systems with timevarying delay, nonlinearity, and Markovian switching. IEEE Transactions on Automatic Control, 50(2),(2005), 217-222.

[30] Zohrabi N., Momeni H. and Abolmasoumi A..Comments on "Delayed-state-feedback exponential stabilization for uncertain Markovian jump systems with mode-dependent time-varying state delays". Nonlinear Dynamics, 73(3), (2013), 1493-1494.

\section{Biography}

Dezhi Liu,received his B.S. degree in Qufu Normal University, China in June 2004 and his M.S.degree in Mathematics from Central South University, China in May 2007. He is currently working towards his Ph.D. degree in Control Engineering at Nanjing University of Science and Technology, China. His current research interest includes stochastic analysis and stochastic control system. 